\title{
The Cognitive Features of Interface Language and User Language
}

\author{
Xi Luo,Lei Di,Xiaohui Zou \\ *Shanghai Shenyue Software Technology Co., Ltd. \\ Room B10, North D., Bld. 8, No. 619 Longchang Rd., \\ Shanghai 200090, \\ China \\ m15002126553 1@163.com \\ *Shanghai Shenyue Software Technology Co., Ltd. \\ Room B10, North D., Bld. 8, No. 619 Longchang Rd., \\ Shanghai 200090, \\ China \\ 2093950 @qq. com \\ ** Sino-American Saerle Research Center \\ Tiangongyuan, Paolichuntianpai Building 2, Room 1235, Daxing \\ Beijing 102629 \\ China \\ 949309225@qq. com
}

\begin{abstract}
The purpose of this paper is to analyze the cognitive features of interface language and user language, clarify user requirements, and optimize interface design. The method steps are: First of all, clarify that interface is wellknown as a display form that users can perceive through their own vision, hearing, operation, etc., as well as a direct way for interaction between software and users. Furthermore, to analyze the interface design and the multi-language features inherent in the user interface, the user language that needs to be converted at the first level is the user's personalized language. In general, it is necessary to understand what the user's inner true thoughts want; the second level of the user language that needs to be converted is the personalized interface that the user wants. However, at this time, the user's requirements are described by combining natural language, graphics, and even metaphor. The result is: Through the dual conversion from user language to interface language, the combination between user requirements and interface design has been clearly defined. At least, the requirements focus has been identified, like the difference between main and auxiliary functions. Grasp this point, a targeted and concise interface design is possible. Its significance lies in: It is not only conducive to the optimization of the specific software interface function design, but also helps people to further discuss the cognitive features of the interface language and the user language in theory.
\end{abstract}

KEYWORDS: interface; user language; interface language

adfa, p. 1, 2011

(C) Springer-Verlag Berlin Heidelberg 2011 


\section{Introduction}

The interface is not strange to most people. Whenever you touch a new piece of software, the first time the user interacts is the interface. Through the operation of the interface, user could achieve the purpose of use. But how can each interface highlight its intention? Deliver the using information to the users in which way? This paper will combine the user interface language and user language to explore and try to answer these questions.

\section{Interface language}

In our daily life, the first interaction with software is presumably the interface. A concise and effective operation interface will not only reduce the user's time for learning the operation, but also make the user really appreciate the convenience and ease of use. At present, most of the web interface or APP interface can highlight its existing significance. Just like the current popular software/APP like Taobao, Netease cloud music, WeChat and so on. Each interface is substantial without missing major functional points. Not all needs can be summed up in a natural language sentence. There are also transitions between language and language.

Nowadays there are many excellent interface designs. Some of them are simple and practical, for example WeChat, wallet finance, etc. In terms of interface design, users can understand at a glance how these interfaces are used and what functions they have. Each interface can be said to have its own value. However, in the beginning of the design process, the interface may not be like the final version handed to the users. The interface still needs to be polished with simplicity before being presented to the user. This is the interface language.

First of all, let's explain what interface language is. The interface language is the medium for human-computer communication and interaction. It can help people organize the logic from the visual interface information, complete the interaction with the interface, and ultimately achieve the purpose of use. ("How do you turn complex logic and information into a simple interface experience?" 2017). User can obtain the operating logic of the entire interface only by looking at it. A good interface language is relatively single and complex. The so-called "single" is to tell the user directly through a simple interface language what kind of problems the interface can help user solve. It saves the process of reading information and learning interface by users. The designer's main job is to inform the user of the simplest information and operation path. But why do we say that a good interface language is in another way complex? The interface language is actually the result of conversion based on the background logic and thinking layers. The step "how to present in a simple way" itself is complicated. It's necessary to really understand what the user really wants, so that the focus by the design could be identified. This understanding process is actually the process of understanding the user language. 


\section{User language}

The user language can tell what the user really wants. Most of the user languages are hidden. However, the vast majority of users who come into contact will speak out the so-called needs in most case. But they are all expressed by the user in an external language. For example: Colleague $\mathrm{A}$ in the office proposed to go to lunch together, and colleague B asked what kind of lunch to eat? Colleague A answered casually. Colleague B suggested: Let's eat noodles. Colleague A said: Just eaten yesterday, let's go to eat rice instead. In this case, colleague A could be seen as a user and colleague B as a designer. Only through external languages, users can also tap into potential internal language. Unlike external language, internal language is a selfquestioning or non-verbal language activity ("Internal language", 2013). In actual life, people communicate with each other basically by external languages. External language is the speech process when people communicate with each other. External language consist of verbal and written language. Oral language is what people has to say to others. However, the grammatical structure and logic system of verbal speech is not required to be complete. When users describe their own needs, most of them will use gestures to explain. ("External language", 2016). But sometimes the external language can't express what the user really wants to express. This is the user's internal language. In fact, the internal language and user language have similarities. The internal language is a verbal process that does not play a role in communication, and it is a verbal activity when the individual thinks. Internal language and external language are inextricably linked. At first the internal language is produced, then it is transformed into an external language that needs to be elaborated. However, the transformation of internal language into external language is actually a shift from a brief summary of language. Therefore, sometimes there was such a phenomenon - the user's needs, through a simple understanding, into the actual operation of the interface, but we found that what users really want was not what we did. The root cause of the problem is the transformation from the internal language to the external language. How to solve this problem? These series of transformation process build essentially a tedious and long process.

At first let's consider user's external language. The people-to-people communication mentioned above is basically external language. But the process by which people use language to communicate is called speech. In the terminology, "language" and "speech" are two different concepts. What is the language? In our opinion, language and speech activities cannot be confused; it is only a definite part of speech activity and of course a major part. Language activities are multi-faceted and complex in nature, cover several fields like physical, physical, and psychological at the same time. They also belong to the fields of individuals and the community. ("Ordinary Linguistics Course" 1980) Speech actually refers to people's use of language. Language can be regarded as a tool used by people to think in communication. In the case of language, it is the use of this tool to conduct communication activities. There might exist some minor deviations between the user's language and speech in the process. All the things like sometimes inappropriate words, fast speech, etc. can cause the deviations. How to solve these deviations? A possible solution is the clever usage 
of pictures and images. Take a "Geomking -- junior middle school plane geometry learning software" example, as shown below.
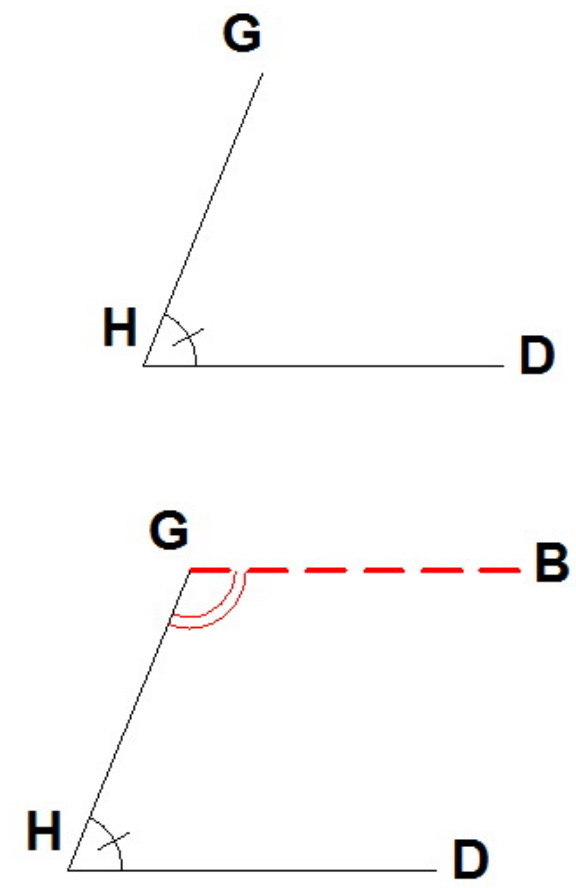

Figure 1. Example graph

This example is to prove that when one of the two intersecting lines is taken as the third straight line and is related to the interior angles on the same side, the basic graphic of the parallel lines related to the interior angles on the same side can be applied or added by the proof. Method: Make a parallel line to the straight line that intersects the third line. (Examples selected from Geomking -- junior middle school plane geometry learning software)

In the Basic Graphic Analysis Method (Fangqu Xu, 1998), each interface is provided with vivid solution steps and graphic transformations. Students and teachers are users, they can see the changes in each step and changing process. It could help them to get a better understanding. That is the graphic-assisted explanation. However, it is not possible that all the words need to be explained in a graphic-assisted manner. There are other ways to give a visual solution. Similar to the example of colleague A and colleague B above, communicate with the user per language to see if it's possible to achieve what the user wants. Repeated communication, excavation at each level, confirmation, from the outside to the inside, so as to achieve the user's real inner needs, that is, the user's own internal language. This is the process by which we learn the internal language of the user from external utterances. After achieved the user's 
internal language, essentially the user's user language, we can then begin to translate the user language into an interface language.

The transformation of user language into interface language is essentially a presentation process from the psychological to the external, together with the multilingual transformation process. The comparison of this group of languages is similar to the internal and external language comparison. The difference is that the logic during the conversion from user language to interface language to is enhanced. Analyzing the user language is a crucial step by the conversion from the user language to interface language. It is related to which information the interface language should pass to the user. Without analyzing the results of the user language, it is impossible to design user-friendly interface. This is also the first difficulty in conversion from user language to interface language - highlighting the key points in the interface language. Most processes of analyzing the user language are silent, and some ideas even disappear. In fact, in this case it's possible to create a simple interface language draft - drawing. The user language could be expressed in the form of drawing. This process could simply the process of conversion into the interface language. Just by the interface language, in fact, there is also opposition - internally and externally. Most users read the interface information from the actual interface, that is, the interface language. With simple thinking logic, interactions can be quickly implemented, which is arguably the external interface language. This is the display of the interface language. Internal interface language is the realization of the interface logic - programming language. Once an interface language does not contain internal contents, it is an interface that can only transmit information and cannot interact. Such an interface could only be used to display. The user can only accept the information given by the interface, no way to interact with the interface. Only combined with programming language, the substantive interface could be presented to the user. The programming language highlights the rigorous logic in the interface language. Which button controls which features, data display, search filters, and so on. These logics cooperate with the interface language to pass the information to the user, after user receives the information transmitted in the interface language and make a relative reaction or operation. If there is no rigorous logic, the possible results are not consistent with what the user wants. This is not an incorrect display but a lack of rigorous logic. Interface language and programming language are also essentially inseparable and mutually dependent. For the user, the user language is internal and hidden; the interface language is visual and can convey information; the programming language is invisible and hidden. However, the concealment of the user language is different from the concealment of the programming language. The user's language is the speech process of the user's heart, and is hidden in thought; and the programming language can be said to be a series of program code written in languages such as java and $\mathrm{C}++$. It is invisible concealment. The entire process of converting from the user language to the interface language is actually interlocking and inseparable. 


\section{Conclusion}

Combining the features of user language and interface language, the process of transformation is a multi-level transformation. The necessary associations exist between each language. Internal language - idea to external language - expression. Understand the user's internal language - idea to user language - requirements. User Language - Requirements to Interface Language - Presentation and Programming Language - Logic. The key is how to achieve the user's internal language and translate it into the real needs of the user. Only by grasping this point you can design a simple and clear interface that the user can understand at a glance what the interface wants to tell the user.

\section{References:}

1. "How to Turn Complex Logic and Information into a Simple Interface Experience?", 2017

2. Selected from http://www.woshipm.com/pd/858699.html This article mainly describes the definition of the interface language and the interface design direction.

3. "External Language", 2016

4. Selected from the Baidu Encyclopedia https://baike.so.com/doc/9324061-9660184.html mainly refers to the definition of the external language. And the difference between internal and external languages.

5. "Internal Language", 2013

6. Selected from the Baidu Encyclopedia https://baike.so.com/doc/6492691-6706401.html Main reference to the definition of the internal language. And the difference between internal and external languages.

7. "General Linguistics Course", 1980

8. Ferdinand de Saussure. Sha Bally, A. Schreiber, and A. Riedlinger co-edited, "General Linguistics Course" by. The Chinese translation was published in 1980.

9. "Geomking -- junior middle school plane geometry learning software",2007--

10. Selected from shanghai Shenyue Software Technology Ltd. http://www.001jihe.net/Home/ShowBig2Index. The software mainly uses the pattern of graphic analysis to help teachers explain the problems of geometry and help students complete geometry exercises.

11. "Basic Graphic Analysis Method",1998

12. Fangqu Xu, Elephant Publishing House, 1998 